\title{
Competitive gene flow does not necessarily maximize the genetic gain of genomic breeding programs in the presence of genotype-by-environment interaction
}

\author{
L. Cao, ${ }^{1 *} \odot$ H. A. Mulder, ${ }^{2} \odot$ H. Liu, ${ }^{1} \odot$ H. M. Nielsen, ${ }^{1} \odot$ and A. C. Sørensen ${ }^{1,3}$ \\ ${ }^{1}$ Center for Quantitative Genetics and Genomics, Aarhus University, Blichers Alle 20, 8830 Tjele, Denmark \\ ${ }^{2}$ Animal Breeding and Genomics Group, Wageningen University and Research, $6700 \mathrm{AH}$ Wageningen, the Netherlands \\ ${ }^{3}$ Danish Pig Research Centre, SEGES, Axeltorv 3, 1609 Copenhagen V, Denmark
}

\begin{abstract}
National and international across-population selection is often recommended and fairly common in the current breeding practice of dairy cattle, with the primary aims to increase genetic gain and genetic variability. The aim of this study was to test the hypothesis that the strategy of truncation selection of sires across populations [i.e., competitive gene flow strategy (CGF)] may not necessarily maximize genetic gain in the long term in the presence of genotype-by-environment interaction $(\mathrm{G} \times \mathrm{E})$. Two alternative strategies used to be compared with CGF were forced gene flow (FGF) strategies, with 10 or $50 \%$ of domestic dams forced to be mated with foreign sires (FGF10\%, FGF50\%). Two equal-size populations $\left(\mathrm{N}_{\mathrm{dams}}=1,000\right)$ that were selected for the same breeding goal trait $\left(\mathrm{h}^{2}=0.3\right)$ under $\mathrm{G} \times \mathrm{E}$ correlation $\left(\mathrm{r}_{\mathrm{g}}\right)$ of either 0.9 or 0.8 were simulated to test these 3 different strategies. Each population first experienced either 5 or 20 differentiation generations $\left(\mathrm{G}_{\mathrm{d}}\right)$, then 15 migration generations. Discrete generations were simulated for simplicity. Each population performed a within-population conventional breeding program during differentiation generations and the 3 across-population sire selection strategies based on joint genomic prediction during migration generations. The $4 \mathrm{G}_{\mathrm{d}_{-}} \mathrm{r}_{\mathrm{g}}$ combinations defined 4 different levels of differentiation degree between the 2 populations at the start of migration. The true rate of inbreeding over the last 10 migration generations in each scenario was constrained at 0.01 to provide a fair basis for comparison of genetic gain across scenarios. Results showed that CGF maximized the genetic gain after 15 migration generations in 5_0.9 combination only, the case of the lowest differentiation degree, with a superiority of $0.4 \%$ (0.04 genetic SD units) over the suboptimal strategy.
\end{abstract}

\footnotetext{
Received October 23, 2020.

Accepted March 15, 2021.

*Corresponding author: cao.lu@qgg.au.dk
}

While in 5_0.8, 20_0.9, and 20_0.8 combinations, 2 FGF strategies had a superiority in genetic gain of 2.3 to $12.5 \%$ (0.21-1.07 genetic SD units) over CGF after 15 migration generations, especially FGF50\%. The superiority of FGF strategies over CGF was that they alleviated inbreeding, introduced new genetic variance in the early migration period, and improved accuracy in the entire migration period. Therefore, we concluded that CGF does not necessarily maximize the genetic gain of across-population genomic breeding programs given moderate $\mathrm{G} \times \mathrm{E}$. The across-population selection strategy remains to be optimized to maximize genetic gain.

Key words: breeding scheme, stochastic simulation, across-population selection, joint genomic prediction

\section{INTRODUCTION}

Introgression of genetic materials from a foreign population to a domestic population (i.e., gene flow) changes the composition of the gene pool of the domestic population when the foreign population is genetically distinct from the domestic population. Gene flow is an extremely important and useful tool for the management of genetic diversity, because it may supplement those endangered or extinct alleles in the course of evolution and consequently help to recover or even increase the genetic diversity of the domestic population (Frankham et al., 2002). Even quite small amounts of gene flow is already capable of preventing much differentiation of subpopulations due to random genetic drift and inbreeding (Falconer and Mackay, 1996). Therefore, gene flow can be used to slow down or arrest the differentiation of domestic populations, alleviate inbreeding, and potentially increase long-term genetic gain.

Slagboom et al. (2019) and Cao et al. (2020) proved the benefits of gene flow on improving genetic gain and alleviating inbreeding by the means of across-population selection of sires based on joint genetic evaluation 
in the presence of genotype-by-environment interaction $(\mathbf{G} \times \mathbf{E})$. Cao et al. (2020) demonstrated that genomic breeding programs could better use these benefits than conventional pedigree-based breeding programs. However, these studies only conducted truncation selection of sires across the domestic and the foreign population. This selection strategy was named as competitive gene flow strategy (CGF) in this study, because it preferred those competitive bulls with highest EBV, regardless of their origin. Characterized by selecting individuals with the highest EBV, CGF prioritized genetic gain at all costs without considering the risk of inbreeding and loss of genetic variance (Cao et al., 2020). Hence, we hypothesized that CGF may not maximize the long-term genetic gain of across-population genomic breeding programs in the presence of $\mathrm{G} \times \mathrm{E}$ although it can maximize genetic gain for a short period (Kimura and Crow, 1978; Henryon et al., 2015), because those selected competitive individuals may not contribute to increasing genetic variability.

To test the hypothesis, we created the opposite alternatives to CGF by introducing forced gene flow strategies (FGF), which forced a fraction of domestic dams to be mated with foreign sires. We stochastically simulated 2 populations of equal sizes in different environments, each with their own breeding program. Apart from varying the across-population selection strategy of sires, we also varied the level of the differentiation degree between the domestic and the foreign population before the across-population selection of sires. The reason of considering these 2 aspects as variables is that selection strategy is fairly controllable for the farmers in practice, and the differentiation degree between populations is a property of the relationship between 2 populations that is hardly constant and nearly impossible to be altered by the farmers. Our assessment on the performance of different selection strategies was from the perspective of the famers in the domestic population.

\section{MATERIALS AND METHODS}

\section{Design}

To compare the performance of FGF and CGF on genetic gain for genomic breeding programs allowing for gene flow across populations in the presence of $\mathrm{G} \times \mathrm{E}$, we simulated 2 dairy cattle populations in different environments, each with their own breeding program. These 2 populations had equal sizes with 1,000 out of 1,500 females selected in each generation. Both breeding programs selected for the same single trait (that can be also regarded as a total merit index), but to simulate $\mathrm{G} \times \mathrm{E}$, the genetic correlation $\left(\mathbf{r}_{\mathrm{g}}\right)$ between the performances of that trait expressed in 2 environments was set as 0.8 or 0.9 . Discrete generations, meaning that breeding animals reproduce just one breeding season, were modeled. Each breeding program consisted of 2 phases, differentiation generations and migration generations. The number of differentiation generations $\left(\mathbf{G}_{\mathbf{d}}\right)$ was 5 or 20 , whereas the number of migration generations was fixed at 15 . The 2 populations were completely isolated during differentiation generations, whereafter across-population selection of sires was allowed in each population during migration generations. Four combinations of $\mathrm{G}_{\mathrm{d}}$ and $\mathrm{r}_{\mathrm{g}}\left(5 \_0.8,5 \_0.9,20 \_0.8\right.$, and 20_0.9) were used to define 4 varying levels of the differentiation degree between the domestic and the foreign population before migration generations. The domestic population referred to the population that received migrant sires, whereas the foreign population referred to the population that donated migrant sires. Each population was the domestic population in terms of the goal trait under selection by its own breeding program, and at the same time the foreign population in terms of the goal trait under selection by the breeding program of the other population. Therefore, there were 2 pairs of domestic and foreign population in this study, each pair of which focused on the goal trait selected by the domestic population.

The selected goal trait was moderately heritable with a constant $\mathrm{h}^{2}$ of 0.3 and an initial genetic mean of 0 in each population. It was controlled by 2,000 biallelic QTL distributed across 30 chromosomes. The additive variance of all QTL explained all the genetic variance of that trait, the initial value of which was 1 . Only females expressed phenotypes for the goal trait. The selection criteria of breeding animals were EBV of the selected trait using within-population pedigree-based BLUP (PBLUP) during differentiation generations and across-population single-step genomic BLUP (ssGBLUP) during migration generations. Dams during both differentiation and migration generations and sires during differentiation generations were truncation selected within populations. Sires during migration generations were selected using FGF50\%, FGF10\%, and CGF sire selection strategy.

FGF50\%. In every migration generation, each breeding program truncation selected 25 foreign males to equally mate with $50 \%$ of the selected domestic females $\left(\mathrm{N}_{\mathrm{dam}}=500,20\right.$ matings per foreign sire $)$ and a constant number of domestic males to equally mate with the remaining selected domestic females $\left(\mathrm{N}_{\text {dam }}=\right.$ 500).

$\boldsymbol{F G F 1 0 \% . ~ I n ~ e v e r y ~ m i g r a t i o n ~ g e n e r a t i o n , ~ e a c h ~}$ breeding program truncation selected 5 foreign males to equally mate with $10 \%$ of the selected domestic fe- 
Table 1. The number of selected sires in each migration generation in 12 scenarios

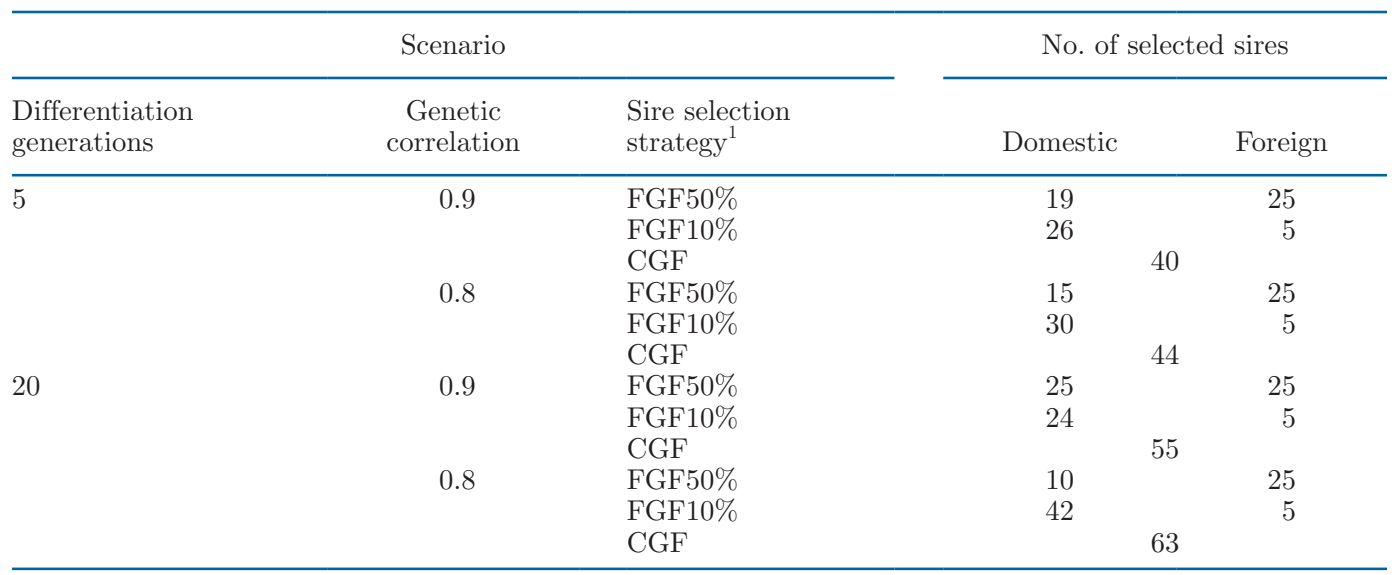

${ }^{1}$ FGF50\% $=$ forced gene flow with $50 \%$ domestic dams mated with foreign sires in each migration generation; FGF $10 \%$ = forced gene flow with 10\% domestic dams mated with foreign sires in each migration generation; $\mathrm{CGF}=$ competitive gene flow.

males $\left(\mathrm{N}_{\mathrm{dam}}=100,20\right.$ matings per foreign sire $)$ and a constant number of domestic males to equally mate with the remaining selected domestic females $\left(\mathrm{N}_{\mathrm{dam}}=\right.$ 900).

$\boldsymbol{C G F}$. In every migration generation, each breeding program truncation selected a constant number of males among the common pool consisting of domestic and external candidates to equally mate with all selected domestic females $\left(\mathrm{N}_{\text {dam }}=1,000\right)$.

The constant number of selected domestic sires in each FGF scenario and the constant number of all selected sires in each CGF scenario was calibrated with the purpose of achieving a rate of true inbreeding during the last 10 migration generations of 0.01 . Table 1 displays the specific number of sires selected in every migration generation in each scenario, via which the number of matings per domestic sire, per foreign sire or per sire can be figured out. The calibration of rate of true inbreeding to a same level was done for a fair comparison of genetic gain among various scenarios. Meanwhile, the calibration of rate of true inbreeding applied only to the last 10 migration generations rather than all migration generations made it possible to observe the difference in true inbreeding among scenarios during the early migration period. How this calibration was obtained is elaborated in Data Analysis in the Inbreeding section. Selected sires were always randomly mated with selected dams according to the allocated number of matings per domestic sire, per foreign sire or per sire. Each dam was only mated with one sire. Each dam delivered 3 offspring each with a probability of 0.5 being male or female, based on the assumption that multiple ovulation and embryo transfer was performed in our design (Thomasen et al., 2020). In total, 12 scenarios were designed using 2 different $G_{d}(5,20)$,
2 different $r_{g}(0.9,0.8)$ and the 3 sire selection strategies (FGF50\%, FGF10\%, CGF) and named in the form of $G_{d \_} r_{g \_}$sire selection strategy. Each scenario was run for 50 replicates.

\section{Simulation Procedures}

The simulation procedures were largely adopted from Cao et al. (2020).

Generation -3,000 to 0: Simulation of Founder Population and Base Population. We first simulated a founder population with an effective population size of 600 throughout 3,000 discrete generations $(t=$ $-3,000 \ldots-1)$ using QMSim software (Sargolzaei and Schenkel, 2009), to construct realistic linkage disequilibrium (LD) information between markers and QTL. The 2,000 QTL and 40,000 SNP markers were kept in the final LD profile. The simulation procedure of founder population was the same as that in Cao et al. (2020) and that for the diverse breeding program tailed for Red dairy cattle in Thomasen et al. (2020). The same founders were used in all replicates of all scenarios. In generation $t=0$, genotypes were randomly sampled from the chromosome pools of founders to establish the base population in each replicate of each scenario. Each base animal was set to be unrelated to each other, and was assigned unique alleles at 6,000 identical-by-decent (IBD) loci to be noninbred. These IBD markers were only used for the purpose of monitoring true inbreeding and were never involved in selection. Phenotyping started with base females, which was computed as the sum of true breeding value (TBV) and residual environmental value. The TBV was calculated as the sum of 2,000 QTL effects. The effects of each QTL on both traits initially followed a bivariate normal distribution 
$N\left(\left[\begin{array}{l}0 \\ 0\end{array}\right],\left[\begin{array}{cc}1 & r_{g} \\ r_{g} & 1\end{array}\right]\right)$ with $r_{g}$ being either 0.9 or 0.8 to model

$\mathrm{G} \times \mathrm{E}$. The initial effects of each QTL were then scaled to enable the additive variances of all QTL for both traits to be 1. The QTL effects stayed unchanged throughout the entire course of simulation, whereas their allele frequencies changed as selection and genetic drift went on. The residual environmental value was always randomly sampled from $\mathrm{N}\left(0,1 / h^{2}-1\right)$. Moreover, how base animals were sampled from the chromosome pools of founders and how alleles at all relevant loci were transmitted from parents to offspring were discussed in Cao et al. (2020).

Generation 1 to $G_{d^{\circ}}$ Differentiation Generations. In generation $t=1$, the parents were obtained by random selection of base males and females. In generations $t=2 \ldots G_{d}$ each breeding program selected parents within its own population based on within-population PBLUP. In the last 5 differentiation generations $t=\left(G_{d}-4\right) \ldots G_{d}$ all selected sires were genotyped to prepare reference information for the implementation of ssGBLUP in subsequent migration generations.

Generation $G_{d}+1$ to $G_{d}+15$ : Migration Generations. During 15 migration generations $t=G_{d}+$ $1 \ldots G_{d}+15$, the 3 sire selection strategies, FGF50\%, FGF10\%, and CGF, were carried out as each breeding program allowed to migrate foreign sires. We adopted across-population ssGBLUP to evaluate the breeding values of all animals and then ranked candidates based on those derived genomic EBV (GEBV). The way of ranking male candidates in FGF scenarios was different form that in CGF scenarios. For instance, in scenario 5_0.9_FGF50\% we ranked domestic and foreign males separately and selected respectively 19 and 25 whoever ranked highest, and so forth in the other FGF scenarios (Table 1). In scenario 5_0.9_CGF, we ranked domestic and foreign males together and selected 40 that ranked highest, and so forth in the other CGF scenarios (Table 1). In all scenarios, we only ranked domestic females to select the 1,000 highest-ranking dams within each population to breed the next generation. All animals were genotyped before selection during migration generations. The simulation was performed using ADAM version 251 (Pedersen et al., 2009). Table 2 summarizes detailed parameter settings used for the simulation of each breeding program.

\section{Genetic Evaluation}

Genetic evaluation was performed in generations $t=$ $2 \ldots G_{d}+15$ using DMU version 6 (Madsen and Jensen, 2013). A bivariate mixed animal model as below was used in each generation:

Table 2. Simulation parameters of each breeding program

\begin{tabular}{ll}
\hline Parameter & Input $^{1}$ \\
\hline Heritability of the selected trait & 0.3 \\
Genetic correlation & $0.9,0.8$ \\
Population size & 1,000 reproductive dams \\
No. of differentiation generations & 5,20 \\
No. of migration generations & 15 \\
Selection criterion during differentiation & Within-population PBLUP-EBV \\
Selection criterion during migration & Across-population ssGBLUP-EBV \\
Selection unit during differentiation (sires) & Within-population \\
Selection unit during migration (sires) & Depend on sire selection strategy \\
Selection strategy during migration (sires) & FGF50\%, FGF10\%, CGF \\
Selection unit (dams) & Within-population \\
Selection method & Truncation selection within selection unit \\
Genotyped (in the last 5 differentiation generations) & Selected sires \\
Genotyped (during migration) & All animals \\
No. of selected sires per differentiation generation & 50 \\
No. of selected sires per migration generation & Depend on scenario (Table 1) \\
No. of selected dams per generation & 1,000 \\
Mating strategy & Random mating \\
No. of matings per sire during differentiation & 20 \\
No. of matings per sire during migration & Depend on scenario \\
No. of matings per dam & 1 \\
No. of offspring per dam & 3 \\
Probability of male and female offspring & $0.5,0.5$ \\
\hline${ }^{1}$ PBLUP = pedigree-based best linear unbiased prediction; ssGBLUP = single-step genomic BLUP; FGF50\% \\
$=$ forced gene flow with 50\% domestic dams mated with foreign sires in each migration generation; FGF10\% \\
= forced gene flow with 10\% domestic dams mated with foreign sires in each migration generation; CGF = \\
competitive gene flow. & \\
& \\
\hline
\end{tabular}




$$
\left[\begin{array}{l}
\mathbf{y}_{1} \\
\mathbf{y}_{2}
\end{array}\right]=\left[\begin{array}{cc}
\mathbf{X}_{1} & 0 \\
0 & \mathbf{X}_{2}
\end{array}\right]\left[\begin{array}{l}
\boldsymbol{\beta}_{1} \\
\boldsymbol{\beta}_{2}
\end{array}\right]+\left[\begin{array}{cc}
\mathbf{Z}_{1} & 0 \\
0 & \mathbf{Z}_{2}
\end{array}\right]\left[\begin{array}{l}
\mathbf{a}_{1} \\
\mathbf{a}_{2}
\end{array}\right]+\left[\begin{array}{l}
\mathbf{e}_{1} \\
\mathbf{e}_{2}
\end{array}\right],
$$

where $\mathbf{y}_{i}$ was the vector of observations of $i$ th trait, $i$ $=1,2 ; \boldsymbol{\beta}_{i}$ was the vector of generations as the fixed effect in the $i$ th environment, the dimension of which was the number of generations to date, i.e., $t ; \mathbf{a}_{i}$ was the vector of additive genetic effects of $i$ th trait; and $\mathbf{e}_{i}$ was the vector of residual effects of $i$ th trait. $\mathbf{X}_{i}$ and $\mathbf{Z}_{i}$ were the incidence matrices connecting $\boldsymbol{\beta}_{i}$ and $\mathbf{a}_{i}$ to $\mathbf{y}_{i}$, respectively. It was assumed

$$
\left[\begin{array}{l}
\mathbf{a}_{1} \\
\mathbf{a}_{2}
\end{array}\right] \sim N\left(\left[\begin{array}{l}
0 \\
0
\end{array}\right],\left[\begin{array}{cc}
\sigma_{a_{1}}^{2} & r_{g} \sigma_{a_{1}} \sigma_{a_{2}} \\
r_{g} \sigma_{a_{1}} \sigma_{a_{2}} & \sigma_{a_{2}}^{2}
\end{array}\right] \otimes \mathbf{H}\right)
$$

and

$$
\left[\begin{array}{l}
\mathbf{e}_{1} \\
\mathbf{e}_{2}
\end{array}\right] \sim N\left([0],\left[\begin{array}{cc}
\sigma_{e_{1}}^{2} & 0 \\
0
\end{array}\right],\left[\begin{array}{l}
\sigma_{e_{2}}^{2}
\end{array}\right] \otimes \mathbf{I}\right)
$$

where $\mathbf{H}$ was the matrix of additive genetic relationships among individuals in the pedigree and $r_{g}=0$ if it was within-population PBLUP in differentiation generations $t=2 \ldots G_{d}$, or the unified genetic relationship matrix of all genotyped and nongenotyped animals in the pedigree (Legarra et al., 2014) and $r_{g}=0.9$ or 0.8 if it was across-population ssGBLUP in migration generations $t=G_{d}+1 \ldots G_{d}+15$; I was the identity matrix; $\otimes$ was the symbol for the Kronecker product of 2 matrices; $\sigma_{a_{i}}^{2}$ was the additive genetic variance of $i$ th trait; $\sigma_{e_{i}}^{2}$ was the residual variance of $i$ th trait (Henderson and Quaas, 1976). For ssGBLUP, the $\mathbf{H}$ matrix requires the genomic covariance matrix of breeding values, $\mathbf{G}$, for genotyped animals, which was obtained by the second method proposed by VanRaden (2008). Residual covariance between 2 traits was zero, because each female who had phenotypes only expressed the breeding goal trait in one environment. The model and its details were largely adopted from Cao et al. (2020).

\section{Data Analysis}

To assess the performance of FGF50\%, FGF10\%, and CGF from the perspective of the famers in domestic populations, the genetic gain, the inbreeding level, the genetic variance, and the accuracy of GEBV of sires within domestic populations were monitored throughout all migration generations, based on a same rate of inbreeding during the last 10 migration generations. We especially analyzed the proportion of foreign sires in all selected sires in each migration generation in all 4 CGF scenarios. Results for each breeding program in each scenario were the averages of 50 replicates. The final results for each scenario were presented as the averages of the results of 2 breeding programs in that scenario, because the 2 breeding programs had equal population sizes and implemented the same selection and mating strategy against each other. The detailed derivation of each result for each breeding program in each replicate was as follows:

Genetic Gain. Genetic gain during the migration period was calculated as the difference between the genetic mean in the last migration generation $t=G_{d}+$ 15 and in the last differentiation generation $t=G_{d}$. The genetic mean in generations $t=G_{d} \ldots G_{d}+15$ was calculated as the mean of TBV of the newborn cohort in generation $t$. The difference in genetic mean between the domestic and the foreign population in the last differentiation generation $t=G_{d}$ was used to measure the differentiation degree between the domestic and the foreign population at the start of the migration period.

Inbreeding. The change of the inbreeding level during the migration period was calculated as the difference between the true inbreeding coefficient in the last migration generation $t=G_{d}+15$ and in the last differentiation generation $t=G_{d}$. The true inbreeding coeffiecient in generations $t=G_{d} \ldots G_{d}+15$ was computed as the mean of the inbreeding coefficents of the 6,000 aforementioned IBD loci in generation $t$, and the inbreeding coefficient of each IBD locus was the average of the probability of the 2 alleles at that locus being IBD over all the newborn cohort in generation $t$. The rate of true inbreeding during the last 10 migration generations was required to be 0.01 to calibrate each scenario. This required rate of true inbreeding was obtained when $1-\exp (b)=0.01$, where $b$ is the regression of the natural logarithm of $(1-$ true inbreeding coefficients) on generation number $t=G_{d}+5 \ldots G_{d}+$ $15, \exp (b)$ is the $b$ power of Euler's number. We obtained this required rate of inbreeding by testing tens of alternative numbers of selected sires until we found the appropriate values listed in Table 1.

Genetic Variance. The genetic variance in generations $t=G_{d} \ldots G_{d}+15$ was computed as the variance of TBV of the newborn cohort in generation $t$. The reduction in genetic variance during the migration period was calculated as the difference between the genetic variance in the last differentiation generation $t=G_{d}$ and in the last migration generation $t=G_{d}+15$.

Selection Accuracy. Selection accuracy of sires in generations $t=G_{d}+1 \ldots G_{d}+15$ was calculated as the Pearson correlation coefficient of TBV and GEBV of 
the male candidates in generation $t$. We evaluated the accuracy of domestic sires and foreign sires separately because the same set of information was of different values to domestic and foreign candidates due to $\mathrm{G} \times \mathrm{E}$. The change in accuracy during the migration period was calculated as the difference between the accuracy in the last migration generation $t=G_{d}+15$ and in the first migration generation $t=G_{d}+1$. The first migration generation $t=G_{d}+1$, rather than the last differentiation generation $t=G_{d}$ as for genetic gain, inbreeding level or genetic variance, was applied as the reference to calculate the change in accuracy of domestic sires, because we could not derive the accuracy of domestic sires in generation $t=G_{d}$ based on the currently available data. However, this replacement had no effect on the conclusions regarding the comparison among the 3 sire selection strategies, because these sire selection strategies came into effect after the genomic prediction in the first migration generation.

Proportion of Foreign Sires in CGF Scenarios. The number of foreign sires using CGF changed during the migration period despite the total number of selected sires was set constant (Table 1) in each migration generation. We calculated the proportion of foreign sires among all selected sires in generation $t=G_{d}+$ $1 \ldots G_{d}+15$ in 4 CGF scenarios.

\section{RESULTS}

\section{Genetic Gain}

In the comparison of 3 sire selection strategies, we found that in all $G_{d \_} r_{g}$ combinations except for $5 \_0.9$ combination, FGF50\% realized the largest genetic gain during migration $\left(\Delta G_{M}\right.$ in Table 3$)$ and the highest eventual genetic mean ( $G_{\text {end }}$ in Table 3 ), followed by FGF10\% and CGF. Specifically compared with CGF, the superiority in $\Delta G_{M}$ of FGF50\% and FGF10\% during migration was absolutely 0.21 to 1.07 genetic standard deviation units and relatively 2.3 to $12.5 \%$ higher than CGF. The genetic trend throughout the migration period (Figure 1) further showed that, FGF50\% and FGF10\% had lower genetic mean than CGF first, but started to have higher genetic mean than CGF from the seventh to tenth and the second to third migration generations, respectively. In 5_0.9 combination, CGF realized the largest $\Delta G_{M}$ and the highest $G_{\text {end }}$ (Table $3)$. Consequently, CGF was slightly superior over the suboptimal FGF50\% in $\Delta G_{M}$, with the absolute superiority of 0.04 standard genetic deviation units and the relative superiority of $0.4 \%$. Hence, the genetic trends using these 3 sire selection strategies in 5_0.9 combination shown in Figure 1 are difficult to distinguish.

Table 3. The comparison of 12 scenarios for the differentiation degree (DD) between the domestic and foreign population at the start of migration period, genetic gain $\left(\Delta G_{M}\right)$, change in inbreeding coefficient $\left(\Delta F_{M}\right)$, and reduction in genetic variance during the migration period $\left(\Delta \sigma_{a M}^{2}\right) ;{ }^{1}$ the genetic mean ${ }^{2}$ in the end of migration period $\left(G_{e n d}\right)^{3}$

\begin{tabular}{|c|c|c|c|c|c|c|c|}
\hline $\begin{array}{l}\text { Differentiation } \\
\text { generations }\end{array}$ & \multicolumn{2}{|l|}{ Scenario } & $\mathrm{DD}$ & $\Delta G_{M}^{5}$ & $G_{\text {end }}^{5}$ & $\Delta F_{M}^{5}$ & $\Delta \sigma_{a M}^{2}$ \\
\hline \multirow{3}{*}{5} & \multirow{2}{*}{0.9} & FGF10\% & \multirow{2}{*}{0.19} & 10.39 & 12.20 & 0.134 & 0.277 \\
\hline & & CGF & & 10.48 & 12.34 & 0.124 & 0.269 \\
\hline & 0.8 & FGF50\% & 0.36 & 9.95 & 11.75 & 0.119 & 0.250 \\
\hline \multirow{5}{*}{20} & \multirow{2}{*}{0.9} & FGF10\% & \multirow{2}{*}{0.81} & 9.24 & 17.37 & 0.104 & 0.253 \\
\hline & & CGF & & 8.58 & 16.79 & 0.110 & 0.247 \\
\hline & \multirow{3}{*}{0.8} & FGF50\% & \multirow{3}{*}{1.61} & 8.72 & 16.87 & 0.062 & 0.155 \\
\hline & & FGF10\% & & 8.43 & 16.68 & 0.107 & 0.164 \\
\hline & & $\mathrm{CGF}$ & & 8.22 & 16.45 & 0.117 & 0.244 \\
\hline
\end{tabular}

${ }^{1} \mathrm{DD}$ was computed as the difference in genetic means between the domestic and foreign population in the last differentiation generation. The higher the value, the more differentiated 2 populations are. $\Delta G_{M}$ and $\Delta F_{M}$ were computed as the difference between the genetic mean and the inbreeding coefficient in the last migration generation and in the last differentiation generation. $\Delta \sigma_{a M}^{2}$ was computed as the difference between the genetic variance in the last differentiation generation and in the last migration generation.

${ }^{2}$ The unit of genetic mean is the genetic SD of the base population.

${ }^{3}$ The end of the migration period refers to the last migration generation.

${ }^{4}$ FGF50\% = forced gene flow with 50\% domestic dams mated with foreign sires in each migration generation; FGF $10 \%=$ forced gene flow with $10 \%$ domestic dams mated with foreign sires in each migration generation; CGF = competitive gene flow.

${ }^{5}$ The SE for $\Delta G_{M}, G_{e n d}, \Delta F_{M}$, and $\Delta \sigma_{a M}^{2}$ were $0.054 \sim 0.093,0.051 \sim 0.100,0.002 \sim 0.005$, and 0.008 0.014, respectively. 


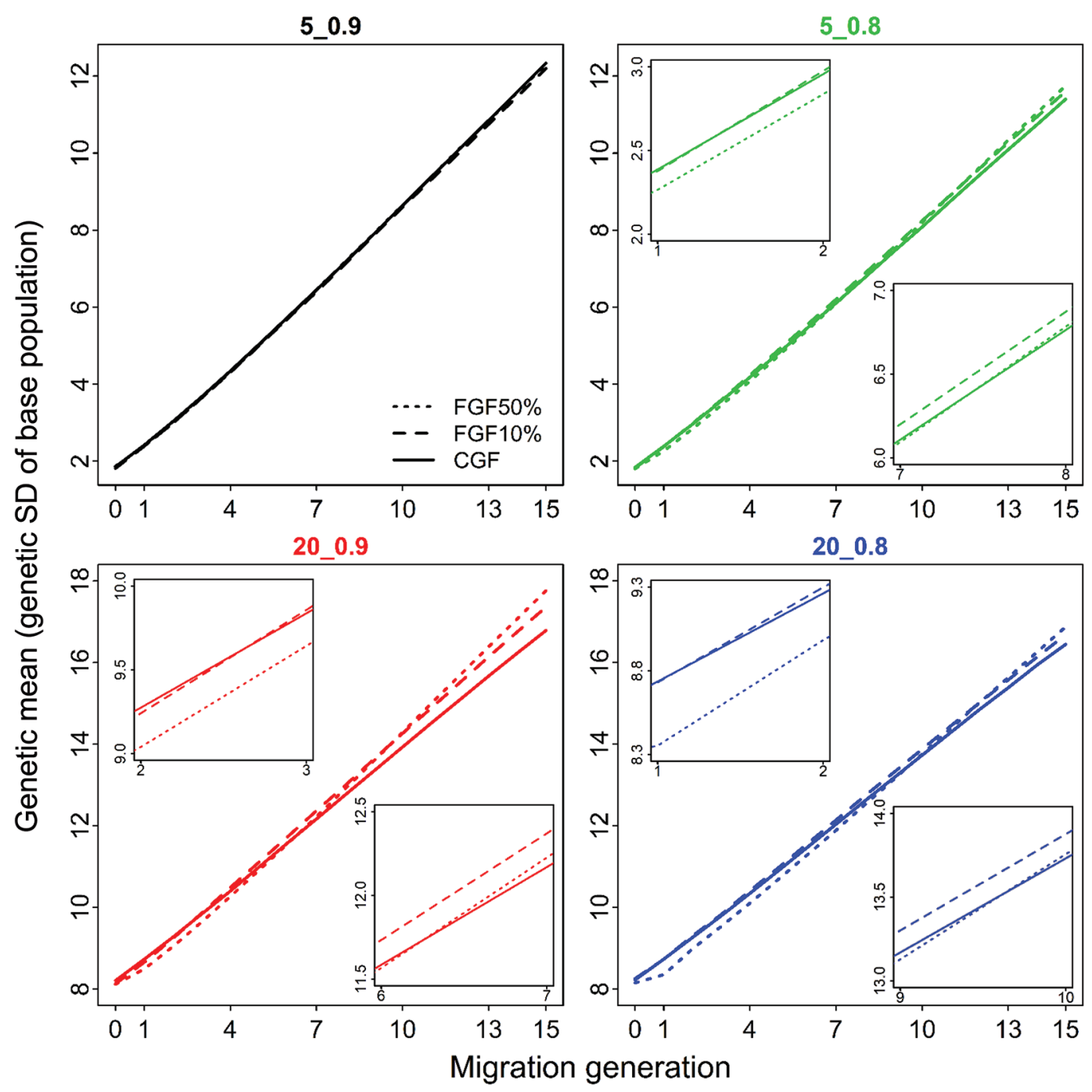

Figure 1. Genetic trend throughout the migration period. Curves in 4 different colors refer to 4 combinations of the number of differentiation generations and the genetic correlation, and curves in 3 different line types refer to 3 sire selection strategies. FGF50\% = forced gene flow with $50 \%$ domestic dams mated with foreign sires in each migration generation; FGF10\% = forced gene flow with $10 \%$ domestic dams mated with foreign sires in each migration generation; $\mathrm{CGF}=$ competitive gene flow. On the horizontal axis, 0 means the last differentiation generation, and 1 and 15, respectively, mean the first and the last migration generation, and so on. We magnified the parts of curves where FGF50\% (bottom right) and FGF10\% (top left) overtake CGF and continuously exceed it thereafter.

Among $4 \quad G_{d-} r_{g}$ combinations, the differentiation degree between the domestic and foreign population at the start of migration period (DD in Table 3 ) increased from 5_0.9, 5_0.8, 20_0.9 to 20_0.8 combination. Not surprisingly, DD increased as $G_{d}$ increased and $r_{g}$ decreased. At the same time, $\Delta G_{M}$ after 15 migration generations decreased as DD increased given the same sire selection strategy (Table 3 ). Note that scenarios with $G_{d}=20$ realized substantially higher $G_{\text {end }}$ compared with scenarios with $G_{d}=5$ simply because of 15 more generations of selection during differentiation period.

\section{Inbreeding Level and Genetic Variance}

The trend of inbreeding coefficient throughout the 15-generations-long migration period varied among 12 scenarios (Figure 2) because we calibrated each scenario for a rate of inbreeding at 0.01 over only the last 10 migration generations. First, the increase in inbreeding coefficient $\left(\Delta F_{M}\right.$ in Table 3$)$ in all scenarios was 0.040 to 0.134 after the whole migration period; FGF50\% always had the lowest $\Delta F_{M}$ especially in combinations with $G_{d}=20$, whereas FGF10\% had a lower or the same $\Delta F_{M}$ than CGF except for in 5_0.9 combination. 

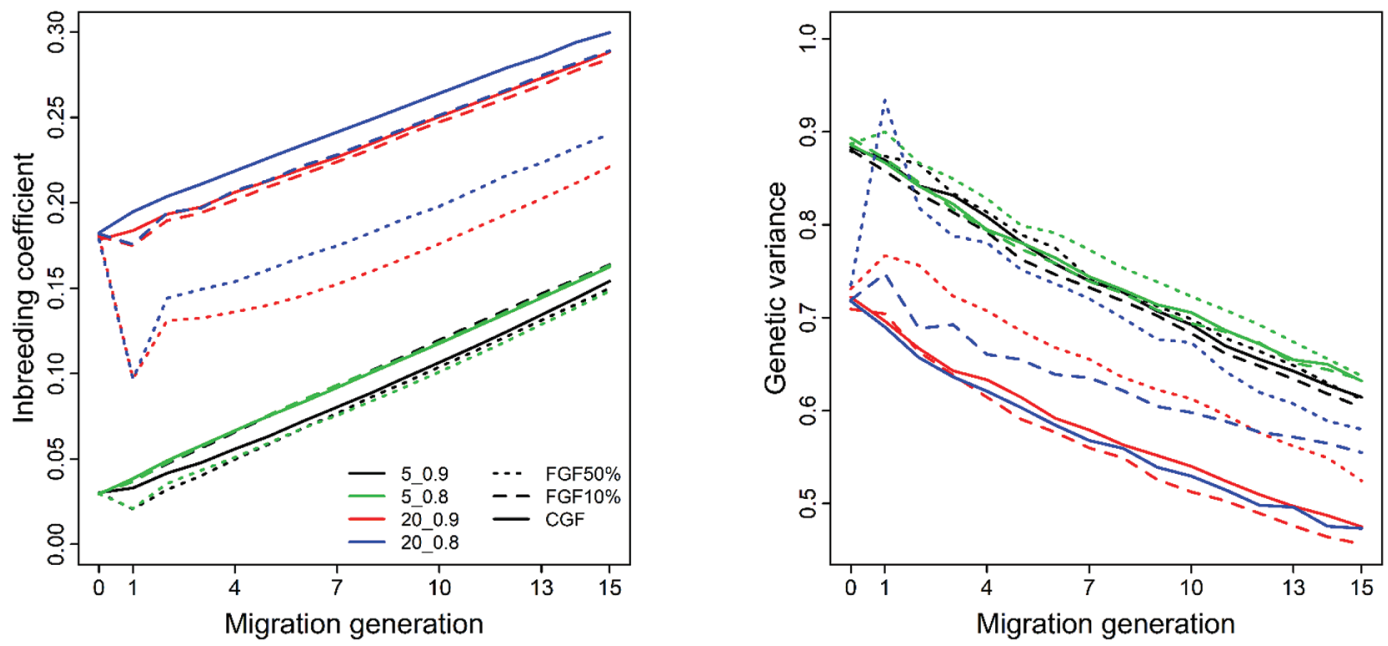

Figure 2. Inbreeding trend (left) and genetic variance (right) throughout the migration period. Curves in 4 different colors refer to 4 combinations of the number of differentiation generations and the genetic correlation, whereas curves in 3 different line types refer to 3 sire selection strategies. FGF50\% = forced gene flow with $50 \%$ domestic dams mated with foreign sires in each migration generation; FGF $10 \%=$ forced gene flow with $10 \%$ domestic dams mated with foreign sires in each migration generation; CGF $=$ competitive gene flow. On the horizontal axis, 0 means the last differentiation generation, and 1 and 15, respectively, mean the first and the last migration generation, and so on.

Second, FGF50\% controlled the inbreeding coefficient to the lowest level in any migration generation within each $G_{d \_} r_{g}$ combination (Figure 2 ). This mainly resulted from FGF50\% reducing the inbreeding coefficient to the lowest level in the first migration generation compared with FGF10\% and CGF, which was particularly obvious in combinations with $G_{d}=20$. The reduction in the inbreeding coefficient in the first migration generation was also observed in FGF10\% scenarios with $G_{d}=$ 20 , although to a smaller degree. The inbreeding coefficient in all CGF scenarios, however, always increased in all migration generations. Note that all the scenarios with $G_{d}=20$ led to a much higher eventual inbreeding coefficient than scenarios with $G_{d}=5$ simply because of 15 more generations of selection during differentiation period.

The reduction of the genetic variance within population of the selected trait was between 0.155 and 0.277 after the whole migration period among all scenarios $\left(\Delta \sigma_{a M}^{2}\right.$ in Table 3$) ; \mathrm{FGF} 50 \%$ had the smallest $\Delta \sigma_{a M}^{2}$ within each $G_{d}-r_{g}$ combination, and FGF10\% had a smaller $\Delta \sigma_{a M}^{2}$ than CGF in 20_0.8 combination only. Two FGF strategies become increasingly superior over CGF in terms of $\Delta \sigma_{a M}^{2}$ as the differentiation degree between the domestic and the foreign population increased. As a result, FGF50\% had the largest eventual genetic variance in almost all $G_{d \_} r_{g}$ combinations, and the ranking between the eventual genetic variance of FGF10\% and CGF depended on $G_{d} \_r_{g}$ combinations (Figure 2). In the first migration generation, the $2 \mathrm{FGF}$ strategies were observed with a less decreased or even an increased genetic variance compared with CGF in the $G_{d \_} r_{g}$ combinations where they had larger eventual genetic variance than CGF. Particularly in scenario 20_0.8_FGF50\%, the surge of the genetic variance in the first migration generation lifted the genetic variance in the subsequent migration generations to the level close to scenarios with $G_{d}=5$. Furthermore, the overall trend of genetic variance corresponded nearly to the reversed overall trend of inbreeding level. Note that scenarios with $G_{d}=20$ saved less genetic variance eventually than scenarios with $G_{d}=5$ because of 15 more generations of selection during the differentiation period. Also note the variation of genetic variance in the last differentiation generation among scenarios with the same $G_{d}$, which should be equal to each other, was simply due to the sampling in the course of stochastic simulation.

\section{Accuracy}

In general, accuracy of domestic sires increased by 0.12 to 0.21 and accuracy of foreign sires increased by 0.07 to 0.30 throughout the entire migration period across all scenarios (Table 4); FGF50\% created the largest increase in accuracy of both domestic and foreign sires and was followed by FGF10\% and CGF within each $G_{d \_} r_{g}$ combination except for 5_0.9. In 5_0.9 combination, 3 sire selection strategies yielded nearly the same increase in accuracy of both domestic and foreign sires. The FGF strategies created a larger increase in accuracy of foreign sires than that of domestic sires in all $G_{d \_} r_{g}$ combinations; FGF50\%, in particular, was 
Table 4 . The absolute increases ${ }^{1}$ in accuracy for domestic and foreign sires during migration period

\begin{tabular}{|c|c|c|c|c|c|c|}
\hline \multirow{2}{*}{$\begin{array}{l}G_{d \_} r_{g} \\
\text { combination }^{2}\end{array}$} & \multicolumn{3}{|c|}{ Domestic sires $^{3}$} & \multicolumn{3}{|c|}{ Foreign sires ${ }^{3}$} \\
\hline & FGF50\% & FGF10\% & CGF & FGF50\% & FGF10\% & CGF \\
\hline 5_0.9 & 0.20 & 0.20 & 0.21 & 0.23 & 0.24 & 0.24 \\
\hline 5_0.8 & 0.20 & 0.19 & 0.17 & 0.28 & 0.23 & 0.14 \\
\hline 20_0.9 & 0.17 & 0.16 & 0.13 & 0.21 & 0.20 & 0.12 \\
\hline 20_0.8 & 0.21 & 0.21 & 0.12 & 0.30 & 0.23 & 0.07 \\
\hline
\end{tabular}

${ }^{1}$ These increases were calculated as the difference in accuracy between the last and first migration generations. ${ }^{2} G_{d} \_r_{g}$ combination refers to the combination of the number of differentiation generations and genetic correlation.

${ }^{3}$ The SE for the increase in accuracy for domestic and foreign sires was $0.005 \sim 0.008$ and $0.006 \sim 0.009$, respectively. FGF50\% = forced gene flow with 50\% domestic dams mated with foreign sires in each migration generation; FGF10\% = forced gene flow with $10 \%$ domestic dams mated with foreign sires in each migration generation; $\mathrm{CGF}=$ competitive gene flow.

observed to have gradually increased the accuracy of foreign sires to a similar or even slightly higher level than that of domestic sires in all $G_{d} \_r_{g}$ combinations (Figure 3). Conversely, CGF created a smaller increase in accuracy of foreign sires than that of domestic sires in all $G_{d \_} r_{g}$ combinations except for 5_0.9 (Table 4). For both domestic and foreign sires in almost each migration generation, FGF50\% gained the highest accuracy and was followed by FGF10\% and CGF within each $G_{d \_} r_{g}$ combination except for $5 \_0.9$ (Figure 3). In 5_0.9 combination, no obvious difference in accuracy of both domestic and foreign sires in each migration generation was shown among the 3 sire selection strategies. Note in the second migration generation, 2 FGF strategies exhibited a larger increase in accuracy of both domestic and foreign sires than CGF within each $G_{d} \_r_{g}$ combination except for $5 \_0.9$. Also note the different clustering

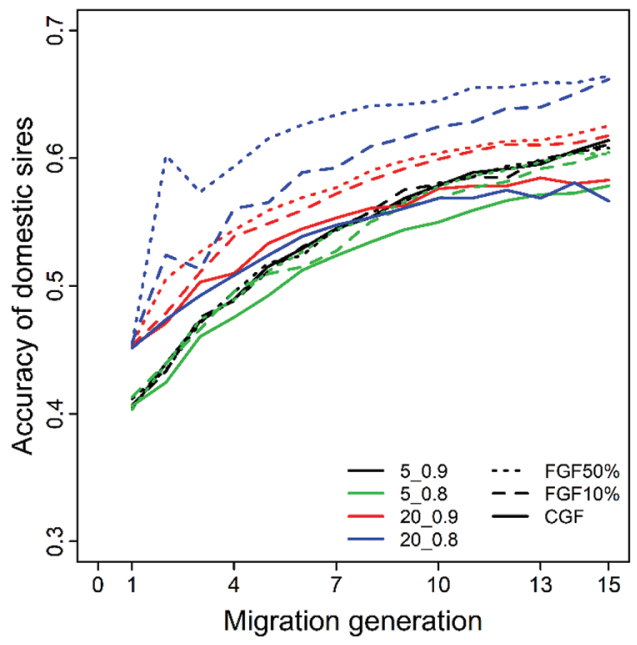

patterns between the accuracy of domestic and foreign sires in the first migration generation: the accuracy of domestic sires in scenarios with same $G_{d}$ was equal, whereas the accuracy of the foreign sires in scenarios with same $G_{d}$ and same $r_{g}$ was equal. With all the aforementioned, it is clear that $5 \_0.9$, the least differentiated $G_{d} r_{g}$ combination, showed distinct patterns from the other $3 G_{d_{-}} r_{g}$ combinations in many ways.

\section{Proportion of Selected Foreign Sires Using CGF}

In total, 40, 44, 55, and 63 sires were selected in each migration generation in 5_0.9_CGF, 5_0.8_CGF, 20_0.9_CGF, and 20_0.8_CGF scenario, respectively (Table 1), with the proportions of foreign sires as shown in Figure 4. The proportion of foreign sires increased in 5_0.9_CGF scenario with a considerable initial value,

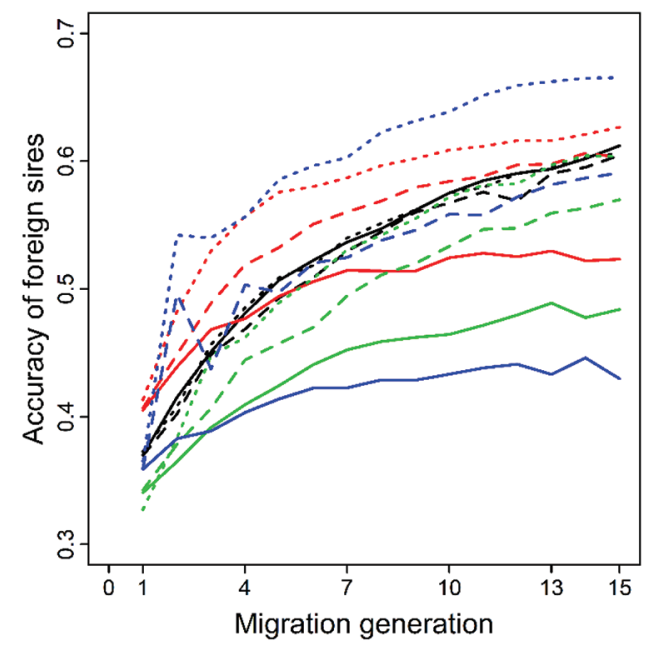

Figure 3. Accuracy of domestic sires (left) and foreign sires (right) throughout the migration period. Curves in 4 different colors refer to 4 combinations of the number of differentiation generations and the genetic correlation, whereas curves in 3 different line types refer to 3 selection strategies of sires. FGF $50 \%=$ forced gene flow with 50\% domestic dams mated with foreign sires in each migration generation; FGF $10 \%=$ forced gene flow with $10 \%$ domestic dams mated with foreign sires in each migration generation; CGF = competitive gene flow. On the horizontal axis, 0 means the last differentiation generation, and 1 and 15, respectively, mean the first and the last migration generation, and so on. 


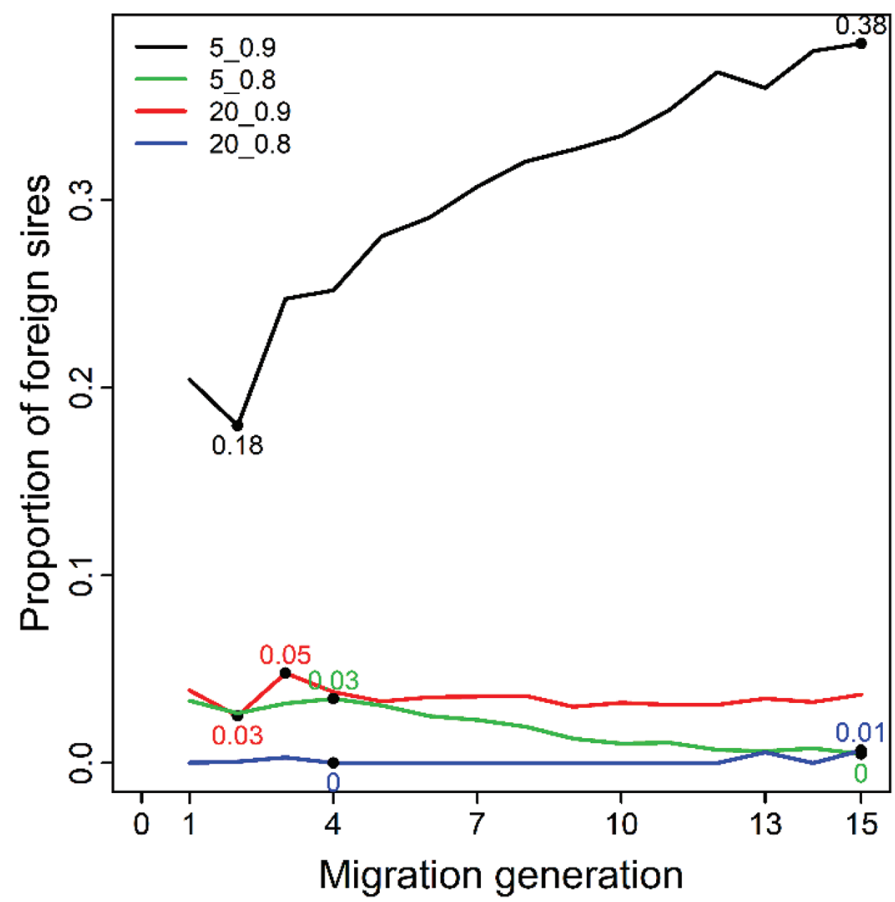

Figure 4. Proportion of foreign sires in all selected sires using competitive gene flow over the whole migration period. Curves in 4 different colors refer to 4 combinations of the number of differentiation generations and genetic correlation. Maximum and minimum values of each curve are marked. On the horizontal axis, 0 means the last differentiation generation, and 1 and 15, respectively, mean the first and the last migration generation, and so on.

decreased to zero in 5_0.8_CGF scenario with a low initial value, and remained stable at a low value in 20_0.9_CGF scenario. In 20_0.8_CGF scenario, CGF selected nearly no foreign sire.

\section{DISCUSSION}

This study confirmed our hypothesis that the selection strategy of CGF does not necessarily maximize the genetic gain of a genomic breeding program that allows gene flow in the presence of $\mathrm{G} \times \mathrm{E}$. This conclusion is more applicable in situations where populations are differentiated above some level. In this paper, 3 $G_{d_{-}} r_{g}$ combinations 5_0.8, 20_0.9, and 20_0.8 exemplified situations with populations differentiated beyond that level, where CGF failed to maximize genetic gain in the comparison with the $2 \mathrm{FGF}$ strategies. In these $3 G_{d_{-}} r_{g}$ combinations, the 2 FGF strategies, compared with CGF, showed a superiority of 0.21 to 1.07 genetic standard deviation units or of 2.3 to $12.5 \%$ in terms of genetic gain after 15 successive generations of migration. This superiority is substantial when it is interpreted under the current Holstein total merit index of 3 Nordic countries (NTM, unit: €), where one standard deviation of the index means 10 units of index value (VikingGenetics, 2020). In fact, this superiority should be even more substantial than the analogy to NTM, given that the standard deviation of EBV is smaller than the standard deviation of TBV, between which the former divided by the latter led to the selection accuracy of the index always less than unity (Falconer and Mackay, 1996). In contrast, both FGF strategies conserved potential for larger long-term genetic gain at the cost of a small quantity of short-term genetic gain, whereas CGF had the advantage of short-term genetic gain but not sufficient to guarantee the long-term genetic gain. The late-developing superiority of FGF in terms of genetic gain by this "sacrifice near to seek far" tactic was attributed to 3 aspects of advantages: alleviating inbreeding and introducing new genetic variance in the early stage of migration, and improving accuracy during the entire migration period.

\section{Advantages in Inbreeding and Genetic Variance}

The FGF strategies decreased the inbreeding level of the domestic population in the first migration generation due to forcibly migrating foreign sires to mate with a certain proportion of domestic dams. Animals in 2 populations were distantly related before migration was allowed, due to the long period of complete isolation during differentiation generations (Szyda et al., 2017). The introduction of foreign sires using FGF strategies reversed the increase of inbreeding for a time and controlled inbreeding at a lower level than the previous generation. In contrast, very few or no foreign sires introduced using CGF led the inbreeding level to increase continuously even though migration was allowed. Honda et al. (2005) had the similar finding in terms of the effect of migration rate of foreign sires on the inbreeding level in the sire lines. The considered migration rates of foreign sires in that study were from 0 to 0.5 with the increment of 0.1 . Therefore, 2 FGF strategies, in particular FGF50\%, were advantageous over CGF in alleviating inbreeding in the early stage of migration. It should be noted that because we expected to observe the difference of inbreeding level in the early stage of migration among 3 selection strategies, we calibrated only the middle and the late stage of migration (i.e., the last 10 migration generations) to the same rate of inbreeding for each scenario. In fact, it does not matter either the whole or part of the migration period was considered in this calibration, because the mechanism by which 2 FGF strategies and CGF strategy operates on inbreeding would not be affected (Kearney et al., 2004; Henryon et al., 2019).

At the same time, the goal trait selected by the domestic population had not been directly selected by 
the foreign population due to $\mathrm{G} \times \mathrm{E}$, so that the foreign population still preserved a larger genetic variation for that trait (i.e., the potential of contributing to increasing the genetic variability of the domestic population). Gustafson et al. (2017) proved the contribution of migrant from a more diverse population to increasing domestic genetic diversity based on their genetic analyses of 2 heterogeneous puma populations. In this study the opportunity of introducing new genetic variability from the foreign population was better seized by FGF strategies, especially FGF50\%, as they guaranteed a considerable contribution from foreign sires to the next generation. Tonteri et al. (2007) found the same rule in aquaculture that the difference in gene flow between populations led to different levels of genetic diversity in populations. Thus, the improvement of genetic variance in the early stage of migration (Alcala et al., 2013), together with the improvement of inbreeding in the early stage of migration, laid a solid foundation for the latedeveloping superiority in genetic gain of FGF strategies over CGF. In addition, the increase of inbreeding level appeared to be one reason to the reduction of genetic variance, in light of the incomplete reversed correspondence between the trend of inbreeding and genetic variance that was observed in each scenario in this study. However, in theory the true cause to the reduction of genetic variance should be genetic drift, which is difficult to distinguish from inbreeding in a finite population (Sørensen, 2005).

\section{Advantage in Accuracy}

Selection accuracy of both domestic and foreign sires increased during the entire migration period due to the growing size of available reference data for ssGBLUP accumulated with time (Casellas and Piedrafita, 2015; van den Berg et al., 2019; Thomasen et al., 2020). The increase in accuracy of both domestic and foreign sires using FGF strategies were larger than those using CGF strategy. In addition, the increase in accuracy of foreign sires were larger than the increase in accuracy of domestic sires using FGF strategies, and vice versa using CGF strategy. These 2 findings revealed that, FGF strategies could better exploit the integrated value of the domestic and foreign data to improve the overall accuracy of the joint genomic prediction during the course of migration, given the same size of joint data set in each scenario. The underlying reason was that FGF strategies built up stronger genetic relationships among candidates in the joint reference population due to the steady exchange of breeding stocks between the 2 populations. Stronger genetic relationship among candidates in the joint reference population has been widely regarded beneficial to converge the LD patterns between the 2 populations and thus, to improve the accuracy of joint genomic prediction (Habier et al., 2010; Zhou et al., 2013; Wientjes et al., 2016). This reason also explained why FGF strategies started to exhibit larger increases in accuracy than CGF in the second migration generation rather than in the first migration generation. In the first migration generation, the genetic relationships between the domestic and the foreign population was not yet established at the time of the genomic prediction that was executed before the selection. Consequently, the accuracy of domestic sires in the first migration generation largely relied on the relevant information of domestic animals in the reference population, which was only affected by $G_{d}$ because larger $G_{d}$ meant larger reference population. On the other hand, the accuracy of foreign sires in the first migration generation largely relied on the relevant information of foreign animals in the reference population, which was not only affected by $G_{d}$ but also by $r_{g}$ due to $\mathrm{G} \times \mathrm{E}$ existing between the performances of the selected trait expressed in the domestic and the foreign environment. Therefore, we observed that the accuracies of domestic sires and of foreign sires in the first migration generation were clustered by $G_{d}$ alone and by $G_{d}$ and $r_{g}$, respectively, in spite of the same set of reference information used by domestic and foreign sires. To summarize, FGF strategies possess the advantage of gaining higher accuracy of joint genomic prediction over CGF in the entire migration period, because FGF can better use the foreign information by establishing and maintaining a solid genetic relationship between populations.

\section{Reranking of Strategies in Case of Low Differentiation Degree}

The 5_0.9 combination, the $G_{d-} r_{g}$ combination with the lowest differentiation degree between 2 populations, exhibited obviously distinct patterns in genetic gain, inbreeding, genetic variance and accuracy among the 3 considered selection strategies compared with the other $3 G_{d \_} r_{g}$ combinations. For example, 5_0.9 combination demonstrated a minimal superiority of CGF regarding genetic gain over the other 2 FGF strategies, whereas the other $3 G_{d \_} r_{g}$ combinations demonstrated a significant superiority of FGF strategies over CGF. It was attributed to a considerable proportion $(0.2 \sim 0.39)$ of domestic dams mated with foreign sires using CGF in 5_0.9, which was intermediate between the proportion using FGF10\% and FGF50\%. The proportion of domestic dams mated with foreign sires using CGF in the other $3 G_{d} \_r_{g}$ combinations was far lower than 
that using FGF10\% and FGF50\%. The proportion of domestic dams mated with foreign sires using CGF was numerically equal to the genetic contribution of foreign sires in this study, because each selected sire irrespective of their origin was assigned an equal number of matings. Therefore, we believe the reranking of genetic gain of 3 selection strategies among $G_{d \_} r_{g}$ combinations indicates the optimal genetic contribution of foreign sires is not always obtainable using CGF or FGF. Instead, we speculate that optimum contribution selection (Meuwissen, 1997; Meuwissen and Sonesson, 1998; Henryon et al., 2015) may always be able to obtain the optimal genetic contribution and thereby realize the largest genetic gain regardless of the differentiation degree. However, this speculation remains to be tested by future investigation.

\section{CONCLUSIONS}

This study has shown that truncation selection of sires across the domestic and the foreign population could only maximize genetic gain for the domestic population in the case of the least differentiation degree between 2 equally sized $\mathrm{G} \times \mathrm{E}$ populations, whereas in the other 3 cases of higher differentiation degree between populations, forcing $50 \%$ domestic dams to be mated with foreign sires maximized the genetic gain. This reranking indicates that we may find an across-population sire selection strategy that can always maximize genetic gain irrespective of the differentiation degree between populations. In other words, the across-population sire selection strategy in the presence of $\mathrm{G} \times \mathrm{E}$ still remains to be optimized.

\section{ACKNOWLEDGMENTS}

We acknowledge GenSAP (Genomic Selection in Animals and Plants) project for providing academic financial support to this study, and CSC (China Scholarship Council) for covering the living stipend of the first author during this study. Mark Henryon (SEGES, Denmark) supported the programming of a key configuration of the used simulation software in this study (ADAM), without which the progress of this simulation study would have been slowed. We are also grateful to Jörn Bennewitz (Hohenheim University, Germany), Flavio Schramm Schenkel (Guelph University, Canada), and Just Jensen (Aarhus University, Denmark) for their constructive suggestions on how to improve the writing of this manuscript. At the same time, we appreciate Morten Kargo (Aarhus University, Denmark) for his efforts to facilitate the completion of this manuscript. The authors have not stated any conflicts of interest.

\section{REFERENCES}

Alcala, N., D. Streit, J. Goudet, and S. Vuilleumier. 2013. Peak and persistent excess of genetic diversity following an abrupt migration increase. Genetics 193:953-971. https://doi.org/10.1534/genetics .112 .147785

Cao, L., H. Liu, H. A. Mulder, M. Henryon, J. R. Thomasen, M. Kargo, and A. C. Sørensen. 2020. Genomic breeding programs realize larger benefits by cooperation in the presence of genotype $\times$ environment interaction than conventional breeding programs. Front. Genet. 11:251. https://doi.org/10.3389/fgene.2020.00251.

Casellas, J., and J. Piedrafita. 2015. Accuracy and expected genetic gain under genetic or genomic evaluation in sheep flocks with different amounts of pedigree, genomic and phenotypic data. Livest. Sci. 182:58-63. https://doi.org/10.1016/j.livsci.2015.10.014.

Falconer, D. S., and T. F. C. Mackay. 1996. Small populations: II. Less simplified conditions. Pages 72-74 in Introduction to Quantitative Genetics. 4. Longman Group.

Frankham, R., J. D. Ballou, and D. A. Briscoe. 2002. Introduction to Conservation Genetics. Cambridge University Press. https://doi .org/10.1017/CBO9780511808999.

Gustafson, K. D., T. W. Vickers, W. M. Boyce, and H. B. Ernest. 2017. A single migrant enhances the genetic diversity of an inbred puma population. R Soc Open Sci. 4:170115. https://doi.org/10 $.1098 /$ rsos. 170115 .

Habier, D., J. Tetens, F. R. Seefried, P. Lichtner, and G. Thaller. 2010. The impact of genetic relationship information on genomic breeding values in German Holstein cattle. Genet. Sel. Evol. 42:5. https: //doi.org/10.1186/1297-9686-42-5.

Henderson, C. R., and R. L. Quaas. 1976. Multiple trait evaluation using relatives' records. J. Anim. Sci. 43:1188-1197. https://doi.org/ 10.2527/jas1976.4361188x.

Henryon, M., H. Liu, P. Berg, G. Su, H. M. Nielsen, G. T. Gebregiwergis, and A. C. Sørensen. 2019. Pedigree relationships to control inbreeding in optimum-contribution selection realise more genetic gain than genomic relationships. Genet. Sel. Evol. 51:39. https:// doi.org/10.1186/s12711-019-0475-5.

Henryon, M., T. Ostersen, B. Ask, A. C. Sørensen, and P. Berg. 2015. Most of the long-term genetic gain from optimum-contribution selection can be realised with restrictions imposed during optimisation. Genet. Sel. Evol. 47:21. https://doi.org/10.1186/s12711 $-015-0107-7$

Honda, T., T. Nomura, and F. Mukai. 2005. Prediction of inbreeding in commercial females maintained by rotational mating with partially isolated sire lines. J. Anim. Breed. Genet. 122:340-348. https://doi.org/10.1111/j.1439-0388.2005.00542.x.

Kearney, J. F., E. Wall, B. Villanueva, and M. P. Coffey. 2004. Inbreeding trends and application of optimized selection in the UK Holstein population. J. Dairy Sci. 87:3503-3509. https://doi.org/ 10.3168/jds.S0022-0302(04)73485-2.

Kimura, M., and J. F. Crow. 1978. Effect of overall phenotypic selection on genetic change at individual loci. Proc. Natl. Acad. Sci. USA 75:6168-6171. https://doi.org/10.1073/pnas.75.12.6168.

Legarra, A., O. F. Christensen, I. Aguilar, and I. Misztal. 2014. Single Step, a general approach for genomic selection. Livest. Sci. 166:5465. https://doi.org/10.1016/j.livsci.2014.04.029.

Madsen. P., and J. Jensen. 2013. A user's guide to DMU, a package for analysing multivariate mixed models. http://dmu.agrsci.dk/ DMU/Doc/Current/dmuv6_guide.5.2.pdf.

Meuwissen, T. H. E. 1997. Maximizing the response of selection with a predefined rate of inbreeding. J. Anim. Sci. 75:934-940. https:// doi.org/10.2527/1997.754934x.

Meuwissen, T. H. E., and A. K. Sonesson. 1998. Maximizing the response of selection with a predefined rate of inbreeding: Overlapping generations. J. Anim. Sci. 76:2575-2583. https://doi.org/10 $.2527 / 1998.76102575 x$

Pedersen, L. D., A. C. Sørensen, M. Henryon, S. Ansari-Mahyari, and P. Berg. 2009. ADAM: A computer program to simulate selective breeding schemes for animals. Livest. Sci. 121:343-344. https://doi .org/10.1016/j.livsci.2008.06.028. 
Sargolzaei, M., and F. S. Schenkel. 2009. QMSim: A large-scale genome simulator for livestock. Bioinformatics 25:680-681. https:// doi.org/10.1093/bioinformatics/btp045.

Slagboom, M., M. Kargo, A. C. Sørensen, J. R. Thomasen, and H. A. Mulder. 2019. Genomic selection improves the possibility of applying multiple breeding programs in different environments. J. Dairy Sci. 102:8197-8209. https://doi.org/10.3168/jds.2018-15939.

Sørensen, A. C. 2005. Aspect of inbreeding in animal breeding. PhD thesis. The Royal Veterinary and Agricultural University.

Szyda, J., T. Suchocki, S. Qanbari, Z. Liu, and H. Simianer. 2017. Assessing the degree of stratification between closely related Holstein-Friesian populations. J. Appl. Genet. 58:521-526. https://doi .org/10.1007/s13353-017-0409-2.

Thomasen, J. R., H. Liu, and A. C. Sørensen. 2020. Genotyping more cows increases genetic gain and reduces rate of true inbreeding in a dairy cattle breeding scheme using female reproductive technologies. J. Dairy Sci. 103:597-606. https://doi.org/10.3168/jds.2019 $-16974$.

Tonteri, A., A. J. Veselov, S. Titov, J. Lumme, and C. R. Primmer. 2007. The effect of migratory behaviour on genetic diversity and population divergence: A comparison of anadromous and freshwater Atlantic salmon Salmo salar. J. Fish Biol. 70:381-398. https:// doi.org/10.1111/j.1095-8649.2007.01519.x.

van den Berg, I., T. H. E. Meuwissen, I. M. MacLeod, and M. E. Goddard. 2019. Predicting the effect of reference population on the accuracy of within, across, and multibreed genomic prediction. J. Dairy Sci. 102:3155-3174. https://doi.org/10.3168/jds.2018-15231.
VanRaden, P. M. 2008. Efficient methods to compute genomic predictions. J. Dairy Sci. 91:4414-4423. https://doi.org/10.3168/jds .2007-0980.

VikingGenetics. 2020. NTM unlocked-complete guide for understanding NTM, our breeds and our breeding g'program. https:/ /innovativebreeding.vikinggenetics.com/brochures/ntm/ntm-full -guide/.

Wientjes, Y. C. J., P. Bijma, R. F. Veerkamp, and M. P. L. Calus. 2016. An equation to predict the accuracy of genomic values by combining data from multiple traits, populations, or environments. Genetics 202:799-823. https://doi.org/10.1534/genetics .115 .183269 .

Zhou, L., X. Ding, Q. Zhang, Y. C. Wang, M. S. Lund, and G. Su. 2013. Consistency of linkage disequilibrium between Chinese and Nordic Holsteins and genomic prediction for Chinese Holsteins using a joint reference population. Genet. Sel. Evol. 45:7. https://doi .org/10.1186/1297-9686-45-7.

\section{ORCIDS}

L. Cao ๑ https://orcid.org/0000-0003-2587-3257

H. A. Mulder ๑ https://orcid.org/0000-0003-2124-4787

H. Liu ( https://orcid.org/0000-0002-0761-326X

H. M. Nielsen ( ) https://orcid.org/0000-0002-8001-5629 